\section{$\underset{\substack{\text { hommes } \\ \text { \& migrations }}}{ }$}

\section{Hommes \& migrations}

Revue française de référence sur les dynamiques

migratoires

$1324 \mid 2019$

Religion et discrimination

\title{
Nous et les autres. Des préjugés au racisme
}

\section{Mikaël Petitjean}

\section{OpenEdition \\ Journals}

\section{Édition électronique}

URL : https://journals.openedition.org/hommesmigrations/8576

DOI : 10.4000/hommesmigrations.8576

ISSN : 2262-3353

\section{Éditeur}

Musée national de l'histoire de l'immigration

\section{Édition imprimée}

Date de publication : 1 janvier 2019

Pagination : 202-205

ISBN : 978-2-919040-44-5

ISSN : $1142-852 X$

\section{Référence électronique}

Mikaël Petitjean, « Nous et les autres. Des préjugés au racisme », Hommes \& migrations [En ligne], 1324 | 2019, mis en ligne le 01 janvier 2019, consulté le 06 janvier 2022. URL : http://

journals.openedition.org/hommesmigrations/8576; DOI : https://doi.org/10.4000/

hommesmigrations.8576 


\section{Initiatives}

\section{Nous et les autres. Des préjugés au racisme}

Entretien avec Elsa Guerry, responsable de la programmation et de l'itinérance des expositions au Musée de l'Homme, et Evelyne Heyer, commissaire scientifique de l'exposition, réalisé par Mikaël Petitjean, chargé de mission Réseau \& Partenariats, Musée national de l'histoire de l'immigration.

Hommes \& Migrations: L'exposition Nous et les autres. Des préjugés au racisme est la première exposition temporaire thématique du Musée de l'Homme depuis sa réouverture en 2015. Pouvez-vous nous indiquer la genèse de ce projet d'exposition?

Elsa Guerry et Evelyne Heyer: Une première exposition inaugurale avait accompagné la réouverture, plutôt axée sur l'historique du projet et la mise en œuvre de la rénovation du musée (Chroniques d'une renaissance). Le thème de la grande exposition thématique sur le racisme a été proposé pendant la rénovation du musée. Il permettait de développer l'un des points évoqués dans le parcours permanent: la diversité biologique de notre espèce à l'époque de la construction scientifique des races. De plus, il permettait de renouer avec la vision universaliste à la base de la fondation du Musée de l'Homme dans les années 1930. Et, enfin, il permettait d'inscrire le Musée de l'Homme comme un musée de société qui aborde les questions d'actualités sous un regard scientifique.

H\&M: L'histoire du Musée de l'Homme est marquée par l'engagement de son fondateur, Paul Rivet, contre le fascisme. Le projet d'exposition Nous et les autres s'inscrit-il dans le prolongement de cet engagement? Quels étaient les objectifs poursuivis par le musée en choisissant le racisme comme sujet d'exposition?

E. G. et E. H.: La volonté de programmer ce sujet a été d'inscrire le musée comme un acteur de l'engagement citoyen et résistant, dans le prolongement des fondateurs du musée et de son directeur de l'époque: Paul Rivet. Ethnologue et directeur du musée, il est l'artisan principal de la nouvelle conception d'un musée comme instrument d'éducation populaire, au service d'une histoire totale de l'Humanité. Produit de son époque, il adopte une vision racialiste des populations. Il s'appuie ainsi sur une démarche naturaliste et classificatoire, héritée du Muséum national d'histoire naturelle, auquel le Musée de l'Homme est rattaché. Tout en refusant la hiérarchisation biologique des «races», il envisage leur différenciation culturelle. Luttant contre l'antisémitisme et le fascisme, s'opposant à la théorie de la «supériorité» d'une prétendue race aryenne, des chercheurs du musée fondent la revue Races et racisme, qui paraît entre 1937 et 1939. Bon nombre d'entre eux affirment cependant l'infériorité des «peuples de couleur». C'est seulement à partir des années 1950 que la notion de « race» est remise en cause par l'Organisation des Nations unies pour l'éducation, la science et la culture (Unesco). Le développement de la génétique des populations permet ensuite d'invalider définitivement le racialisme. Tous les hommes sont les produits de migrations et de métissages: tous sont différents mais parents.

H\&M: En 2017, comment un musée élabore-t-il un discours visant à déconstruire le racisme et donc, in fine, à lutter contre ce dernier ? Cette thématique a-t-elle conduit à un traitement expographique spécifique? Une exposition comme démarche de médiation scientifique est-elle suffisante? Faut-il lui adjoindre une programmation à cette offre muséographique?

E. G. et E. H.: La volonté d'exposer un concept et des idées plutôt que des collections n'est pas anodine et est assez novateur pour le Muséum national d'histoire naturelle. L'idée était de sortir des expositions qui présentent des collections qui incarnent la mise en œuvre du racisme, mais plutôt de déconstruire scientifiquement le concept de racisme en montrant comment cette notion se construit et dans quels contextes. À la croisée des sciences et des sciences humaines, l'objectif était de croiser les différents points de vue scientifiques sur le sujet: de la psychologie sociale, en passant par l'histoire, la génétique ou bien la sociologie.

La scénographie a été pensée dès le départ comme immersive, le cahier des charges 
le demandait. La volonté de la commissaire d'exposition, Anne Stephan, était de proposer au visiteur une expérience de visite en trois étapes: celle de la connaissance, de la conscience, puis du sens. La visite pouvait se faire ainsi en visite libre, le visiteur déambulait dans un parcours immersif et guidé par la succession des dispositifs scénographiques. Des ateliers ont été développés en parallèle des modules de l'exposition. La programmation scientifique et culturelle venait en appui à l'exposition pour explorer des pistes non abordées dans l'espace d'exposition.

\section{H\&M: Quelles furent les réactions des visiteurs devant cette exposition? Quel bilan le Musée de l'Homme en a-t-il retiré?}

E. G. et E. H.: Le bilan a été globalement favorable et très positif. Le planning des visites scolaires a été complet pendant 6 mois (exposition ouverte pendant 9 mois). Plusieurs créneaux de visite ont été ajoutés à quelques mois de la fermeture.

Le public cible était un public large (+ de 10 ans) mais avec une volonté forte de toucher le public adolescent. Les retours des médiateurs sur le sujet ont été très positifs: les adolescents ont montré un véritable intérêt sur le sujet et se sont investis lors des visites en groupe. D'ailleurs, plus de $35 \%$ des visiteurs avaient moins de 25 ans, tant en semaine que pendant le week-end. Conçue dans un contexte complexe (printemps 2015), une certaine forme d'appréhension s'est manifestée avant l'ouverture au sein des équipes de médiation. Une forte préparation a été réalisée auprès des équipes en interne pour préparer des outils de réponse ou pour désamorcer certaines réactions sur le sujet. L'exposition a été relayée dans de nombreux médias sans heurt mettant en avant le parti pris scientifique et non militant développé dans l'exposition.

Plusieurs partenaires extérieurs se sont manifestés en phase de conception de l'exposition intéressés par les questionnements posés par ce type d'exposition: questions de sociétés, questions citoyennes. Cette exposition a permis de développer de nouveaux partenariats avec des institutions muséales non habituées à travailler avec le Muséum national d'histoire naturelle. Le projet est appuyé par l'Unesco et la Délégation interministérielle à la lutte contre le racisme, l'antisémitisme et la haine anti-LGBT (Dilcrah).
H\&M: Le Muséum national d'histoire naturelle, dont dépend le Musée de l'Homme, dispose d'une longue expérience de l'itinérance de ces expositions. Le Musée de l'Homme a souhaité travailler sur une version itinérante de l'exposition dès les premières phases de conception de Nous et les autres. Pouvez-vous nous indiquer comment ce projet d'itinérance s'est élaboré et quels objectifs étaient poursuivis?

E. G. et E. H.: Pour répondre à la demande d'itinérance qui s'est faite un an avant l'ouverture, nous avons réfléchi à la façon de valoriser ces données en gardant l'identité de l'exposition tout en l'adaptant aux lieux d'accueil: tant du point de vue de la forme que des contenus. Cette réflexion s'est faite en parallèle d'une réflexion plus large initiée par le service des itinérances du Jardin des plantes qui travaillait déjà à faire évoluer cette offre. Cette solution permet de réduire les coûts en maintenance, logistique et stockage des mobiliers.

La conclusion a été la même: faire circuler l'exposition en version dématérialisée. Ce n'est plus les mobiliers qui circulent (cimaise, écrans, cartels) mais les contenus (textes en deux langues, films, interactifs, collections). Cette nouvelle forme d'itinérance permet de diffuser au plus grand nombre et de travailler avec les équipes tant sur les contenus que sur les mobiliers à disposition. Les lieux d'accueil s'approprient l'exposition. Elle habille les murs, nous adaptons en faisant du «sur-mesure». Certains contenus sont intégrés selon le contexte historique ou scientifique local et les publics habitués à fréquenter le lieu. 


\section{H\&M: Quelles formes scénographiques ont été retenues pour cette itinérance?}

E. G. et E. H.: Plusieurs formes ont été retenues: une version légère, appelée "Petite forme», constituée de 10 panneaux et qui peut être accompagnée des films de l'exposition si la structure d'accueil le souhaite. La version graphique ou scénographique (selon la surface) joue avec l'espace, s'installe dans les murs. Aujourd'hui, elle a été adaptée en différentes versions de $70 \mathrm{~m}^{2}$ à $300 \mathrm{~m}^{2}$. Le service de la programmation et de l'itinérance des expositions accompagne le preneur en adaptant le scénario

(programme muséographique adapté), parfois en coordonnant le suivi de réalisation avec le graphiste ou le scénographe, en veillant à la validation des dispositifs avec la commissaire scientifique du Muséum national d'histoire naturelle.

H\&M: Comment les partenaires accueillant l'exposition ont-ils été associés au projet? Quelles ressources sont-elles mises à leur disposition pour leur permettre de préparer les accrochages de l'exposition en amont?

E. G. et E. H.: Les partenaires sont associés à l'adaptation du 
scénario. Plusieurs réunions et échanges de documents permettent de se mettre d'accord sur la version à mettre en œuvre. Ils prennent en charge financièrement la réalisation des travaux graphiques ou scénographiques. Le Muséum national d'histoire naturelle accorde une cession de droits d'utilisation des contenus sur une durée précise contre rétribution. L'accompagnement muséographique et la coordination du projet font parties de l'offre ainsi que la programmation culturelle et scientifique et les ateliers de médiation. Plusieurs documents sont mis à disposition: un mémoire d'exposition qui permet d'avoir une vision complète de l'exposition dispositif par dispositif, un descriptif détaillé des contenus textes et audiovisuels, un film présentant l'exposition originale, etc.

\section{H\&M : Quels types de lieux accueillent la version itinérante de Nous et les autres?}

E. G. et E. H.: Nous avons jusqu'ici travaillé avec des structures très différentes: le Centre européen des résistants déportés (camp de concentration du Struthof), un lieu citoyen appelé «site départemental de Pierresvives» dans l'Hérault. Ce site fait circuler pendant 2 ans dans tout le département la version petite forme dans les collèges, les lycées et les agences culturelles. Une version adaptée va ouvrir en mars eu Chili au Musée de la mémoire et des droits de l'Homme $\left(150 \mathrm{~m}^{2}\right)$, une autre est en cours de présentation à Valence au Centre du génocide arménien $\left(200 \mathrm{~m}^{2}\right)$, mais également au Musée de biosciences de Laval (Québec, Canada). La petite forme est diffusée via des associations, des acteurs locaux ou des mairies. 